Dear Author,

Please, note that changes made to the HTML content will be added to the article before publication, but are not reflected in this PDF.

Note also that this file should not be used for submitting corrections. 


\title{
Neural differences between monolinguals and early bilinguals in their native language during comprehension
}

\author{
P. Román ${ }^{\mathrm{a}, \mathrm{b}, *}$, J. González a ${ }^{\mathrm{a}}$ N. Ventura-Campos ${ }^{\mathrm{a}}$, A. Rodríguez-Pujadas a , A. Sanjuán ${ }^{\mathrm{a}, \mathrm{c}}$, C. Ávila ${ }^{\mathrm{a}}$ \\ a Department of Basic and Clinical Psychology and Psychobiology, Universitat Jaume I, Castelló 12701, Spain \\ ${ }^{\mathrm{b}}$ Department of Psychology, Pennsylvania State University, University Park 16802, USA \\ ${ }^{c}$ Wellcome Trust Centre for Neuroimaging, University College, London WC1N 3BG, UK
}

\section{A R T I C L E I N F O}

\section{Article history:}

Received 23 December 2014

Revised 15 July 2015

Accepted 20 July 2015

Available online $\mathrm{xxxx}$

\section{Keywords:}

Bilingualism

Sentence comprehension

Native language processing

fMRI

\begin{abstract}
A B S T R A C T
Research has shown that semantic processing of sentences engages more activity in the bilingual compared to the monolingual brain and, more specifically, in the inferior frontal gyrus. The present study aims to extend those results and examines whether semantic and also grammatical sentence processing involve different cerebral structures when testing in the native language. In this regard, highly proficient Spanish/Catalan bilinguals and Spanish monolinguals made grammatical and semantic judgments in Spanish while being scanned. Results showed that both types of judgments recruited more cerebral activity for bilinguals in language-related areas including the superior and middle temporal gyri. Such neural differences co-occurred with similar performance at the behavioral level. Taken together, these data suggest that early bilingualism shapes the brain and cognitive processes in sentence comprehension even in their native language; on the other hand, they indicate that brain over activation in bilinguals is not constrained to a specific area.
\end{abstract}

(c) 2015 Elsevier Inc. All rights reserved.

\section{Introduction}

A substantial proportion of the world population is bilingual and speaks more than one language fluently. Bilingual speakers routinely produce and understand sentences belonging to two (or more) languages without difficulty. Therefore, understanding how two languages coexist in one brain, with little conflict or interference between both codes, is an issue of great theoretical and applied interest.

One important question during the last years has focused on whether a bilingual brain processes linguistic information in the same manner as a monolingual brain. More specifically, there is an ongoing debate as to whether an early and/or continued exposure to more than one language yields changes in the pattern of brain activity during language processing. It has been extensively demonstrated that many early childhood experiences can permanently influence brain organization (Fine, Finney, Boynton, \& Dobkins, 2005; Neville \& Bavelier, 2001; Ohnishi et al., 2001; Petersson, Reis, Askelof, Castro-Caldas, \& Ingvar, 2000) and in the linguistic domain, some early experiences result in persistent behavioral and neurological changes (Lenneberg, 1967; Mayberry

* Corresponding author at: Department of Psychology, The Pennsylvania State University, 111 Moore Building, University Park, PA, USA.

E-mail address: per3@psu.edu (P. Román).
\& Eichen, 1991; Neville et al., 1997; Newman, Bavelier, Corina, Jezzard, \& Neville, 2002; Petitto et al., 2000; Roder, Stock, Bien, Neville, \& Rosler, 2002). The early acquisition of a second language seems to play an important role in neural organization regardless of the level of proficiency (Pakulak \& Neville, 2011) and an increasing number of neuroimaging studies in bilingualism have shown that also late experience with more than one language lead to structural and functional modifications in the brain (Kroll, Bobb, \& Hoshino, 2014).

Functional differences in the brain between bilinguals and monolinguals have been observed in word comprehension and production. Rodríguez-Fornells and colleagues observed increased activation in the left inferior frontal gyrus (IIFG) and superior temporal cortex (STC) in Spanish-Catalan bilinguals compared to monolinguals in a lexical decision task (Rodríguez-Fornells, Rotte, Heinze, Nösselt, \& Münte, 2002). However, a study by Parker-Jones et al. showed increased brain activation for bilinguals compared to monolinguals in tasks requiring speech production (reading or naming) but not during the semantic decision tasks in either their native or in their second language (Parker-Jones et al., 2012). Specifically, these authors observed a higher blood oxygen level dependent (BOLD) signal response for bilinguals in the IIFG (pars triangularis and opercularis), superior temporal gyrus (STG), planum temporale and dorsal precentral gyrus when a task required retrieving and articulating words in both their 
native and second language (L2). Since this group of regions is classically associated with language processing, they considered that increased recruitment in bilinguals arose because word retrieval is more demanding due to the co-activation of two languages. Palomar-García et al. (2015) reached a similar conclusion. These authors mirrored the production-only differences in a study in which early balanced bilinguals of Spanish/Catalan and Spanish monolinguals carried out listening and naming tasks in their native language. However, cerebral regions that mediated production in bilinguals were posterior (right STG and posterior cingulate cortex). It is important to highlight three aspects of their study that should maximize similarities in cognitive and neural processing between bilinguals and monolinguals: (1) the sample in their study was early balanced bilinguals; (2) their participants were tested in one language only, therefore reducing the need of language co-activation (e.g. Elston-Gütler, Gunter, \& Kotz, 2005); and, finally, (3) the language of testing was their native language. Despite all this, bilinguals and monolinguals presented a different pattern of neural activation.

Also, studies on sentence comprehension have observed differences between bilinguals and monolinguals in the brain. Kovelman, Baker, and Petitto (2008) performed an fMRI study comparing brain activations of English monolinguals and early Spanish/English bilinguals. Participants had to judge whether visually presented sentences were plausible or not. The sentences varied in their syntactic complexity and were presented in each language in separated blocks for bilingual speakers. For monolinguals, only English sentences were presented. Results showed that bilinguals and monolinguals yielded similar speed and accuracy at the behavioral level, but their brain activations presented some differences in English sentence processing. Neuroimaging analyses revealed that bilinguals had - similar to word processing research - a significantly greater activation in the IIFG (particularly within BA 44/45) than monolinguals. According to Kovelman and colleagues, this activation difference in the IIFG provided evidence suggesting a possible "neural signature" for bilinguals as a consequence of an early exposure to two languages. On the other hand, a greater activation of some cortical areas in the frontal lobe could be indicative that sentence processing in highly proficient bilingual speakers is more effortful for them than for monolingual speakers (Bialystok, Craik, Green, \& Gollan, 2009), since some regions in the frontal cortex are associated with cognitive control (i.e. conflict monitoring, interference resolution, and selection of information when both languages are co-activated). In a recent fNIRS study using the same task and materials as Kovelman et al., Jasinska and Petitto (2013) observed that differences between bilingual and monolingual adults appeared in the right STG, but not in the IFG regardless the type of sentence.

While Kovelman et al. and Jasinska and Petitto used semantic judgment tasks, Wartenburger and collaborators used two types of tasks on sentences in an fMRI study with Italian-German bilinguals: one based on a semantic judgment and one based on a grammatical judgment. They found quite a different pattern of brain activity depending on the nature of the task (Wartenburger et al., 2003). Participants were bilinguals with a variable age of acquisition and variable proficiency level of their L2. Data showed that brain organization underlying semantic processing is more influenced by proficiency level in L2, whereas age of L2 acquisition had a more pronounced effect on the neural representation of grammatical processes. Indeed, Wartenburger et al.'s fMRI study was the first to demonstrate that age of L2 acquisition is crucial for grammatical processing (Perani \& Abutalebi, 2005). However, since they did not include a sample of monolingual speakers, this study could not address the question of whether or not bilingual and monolingual brain activations differ during grammatical processing. Rüschemeyer, Fiebach, Kempe, and Friederici (2005) did compare native speakers of German to late learners. They presented auditory sentences that were semantically or syntactically violated and non-violated. Syntactically correct and incorrect sentences elicited increased activation in the pars opercularis of the IFG and bilaterally in the head of the caudate in late L2 learners compared to native speakers. No differences appeared in the semantic condition. This pattern is similar to event-related potentials (ERPs) research (e.g. Ojima, Nakata, \& Kakigi, 2005), in which highly proficient late L2 learners show native-like ERPs in response to semantic information (a negativity around $400 \mathrm{~ms}$ or N400) but differences in syntactic processing (absence of a left anterior negativity [LAN] in the complex LAN/P600).

To sum up, there is evidence that semantic processing presents a native-like neural pattern in late highly proficient bilinguals, when in contrast grammatical processing in bilinguals entails differences compared to monolinguals. Since Wartenburger et al. found that age of acquisition has a larger impact on grammatical rather than semantic processing, it is particularly important to explore how early bilingualism affects sentence processing compared to monolinguals. Research indicates that differences in neural patterns between monolinguals and bilinguals are modulated not only by age of acquisition (Jasinska \& Petitto, 2013) but, as explained above, by those conditions that minimize interference between languages (e.g. monolingual context, processing of the native language, etc.; Palomar-García et al., 2015). Therefore, in the present work, we explore neural differences between bilinguals and monolinguals for semantic and grammatical processing in a native-like setting; monolinguals are compared to bilinguals that: (1) have acquired their second language early in life, (2) are balanced in both languages, (3) are tested only in their native, dominant language and, therefore, the experimental setting is monolingual; and, finally, (4) we use simple sentences. The use of simple sentences is important for our goal in two ways. On one hand, complex sentences (as those used in Kovelman et al.'s and Jasinska \& Petitto, 2013) involve increased cognitive control demands (Ye \& Zhou, 2009). Since executive control functions/engagement seem to differ between monolinguals and bilinguals in linguistic (e.g. Rodríguez-Fornells, de Diego Balaguer, \& Münte, 2006) and non-linguistic tasks (e.g. Colzato et al., 2008), even under similar behavioral performance (Rodríguez-Pujadas et al., 2013), the recruitment of executive control might be a crucial difference between monolinguals and bilinguals in language processing (Abutalebi, 2008). In terms of neural substrates, syntactic complexity and increased demands of executive control are both related to changes in the IFG (Fiebach, Vos, \& Friederici, 2004; Just, Carpenter, Keller, Eddy, \& Thulborn, 1996). It is desirable, thus, to lessen differences in terms of the cognitive demands that a task places on each group to reveal differences in linguistic processing per se. On the other hand, simple sentences are acquired earlier in life (see Clark, 2009) and consequently processing of simple sentences may be more prone to show steady changes in terms of neural markers. Clahsen and Felser (2006), for example, stated that although the native language and L2 processing can become more similar, however, even highly proficient bilinguals present differences in processing complex syntax. Friederici, Steinhauer, and Pfeifer (2002) suggested that in using artificial languages, late learners could use native-like neural correlates in settings with few rules and words, which resemble simple sentences (Pakulak \& Neville, 2011). Taken into consideration simultaneous bilinguals (early learners), research shows that they are exposed to a lesser extent to each of their languages in relation to monolinguals whether referring to the lexicon (Bialystok, Luk, Peets, \& Yang, 2010), or syntactical structures. This is because they are in contact with an increased diversity of words for each concept and with a diversity of linguistic structures (MacLeod, Fabiano-Smith, Boegner-Pagé, \& Fontolliet, 2013). The use of simple sentences 
helps to equate monolinguals and bilinguals and to reveal a true neural signature of bilingualism in linguistic processing.

Under these circumstances, we want to test the existence of a neural signature of bilingualism in language processing in general, extending results from research in semantic processing (Kovelman, Shalinsky, Berens, \& Petitto, 2008; Kovelman, Baker, et al., 2008) and to understand the nature of such differences.

With respect to our predictions, we have to consider the evidence reviewed above. At the brain level, studies in word produc tion-comprehension and sentence comprehension found differences in IFG and/or STG activation between bilinguals and monolinguals (Jasinska \& Petitto and Kovelman et al. in semantic processing in their native language and L2 respectively; and Rüschemeyer et al. in syntactic processing in L2). Critically, such dissimilarities in recruitment of IFG and STG may vary in terms of age of acquisition (Wartenburger et al., 2003); type of processing, being more related to production and specifically to phonological and pre-lexical activation in production (Rodríguez-Fornells et al., 2002; Rüschemeyer et al., 2005; Parker-Jones et al., 2012); and language context (monolingual contexts and tested in the native language; Palomar-García et al., 2015). Accordingly, we expect early balanced bilinguals and monolinguals to be more alike when they are tested in their native language, whether they are processing semantic or grammatical violations.

\section{Methods}

\subsection{Participants}

Forty-three participants (23 females) from the bilingual region of Castellon (Spain) took part in our study in exchange for a small honorarium. All participants were right handed as judged by the Edinburgh Handedness Inventory (Oldfield, 1971) and had normal or corrected to normal visual acuity. None of them reported neurological or psychiatric disorders. Twenty-three participants (11 females) were Spanish/Catalan bilinguals, and 20 (12 females) Spanish monolinguals.

They completed a subjective language experience questionnaire that provided information about their language history in both Catalan and Spanish. The questionnaire included two rating scales. The first one asked questions regarding the frequency of use of each language $(1=$ only Spanish, $7=$ only Catalan). It comprised different stages in life and different contexts: before primary school, primary and secondary school (at home, school and free time), and adulthood (at home, university, work, and free time). The second scale measured self-reported proficiency and consisted of a four-point scale ( 1 = non-proficient; 4 = very proficient) with which participants evaluated their ability in Catalan and Spanish in the following domains: reading, listening, speaking, and writing. Both the subjective language experience and the self-reported proficiency questionnaires were administered in prior studies (Garbin et al., 2010, 2011). Spanish was the native language for both groups that showed comparable level of proficiency ( $t$-tests comparing the two groups in the four mentioned dimensions of Spanish, $p s>.05$ ). The bilingual group reported a percentage of use of $50 \%$ and $66.95 \%$ for listening and speaking in L2. The monolingual group reported a percentage of use of $9.25 \%$ and $0 \%$ for listening and speaking in L2. Table 1 summarizes results collected from language experience and proficiency measures.

\subsection{Material}

One hundred and twenty simple sentences in Spanish composed the stimulus material in the following manner: 40 sentences were grammatical and semantically correct, 40 included a grammatical violation, and 40 included a semantic violation. The grammatical violation consisted of disagreements of number (either noun-verb or article-noun disagreement) or gender (articlenoun), e.g. "El pez nadan en el río" (the fish swim in the river). The semantically incorrect sentences were grammatically correct but presented semantic violations such as "La piedra flota en el agua" (the stone floats on water). The sentences did not differ between conditions in length (number of characters; $\quad M_{\text {correct }}=28.45, \quad \mathrm{SD}_{\text {correct }}=3.15 ; \quad M_{\text {grammatical }}=27.92$, $\left.\mathrm{SD}_{\text {grammatical }}=3.33 ; M_{\text {semantic }}=28.13, \mathrm{SD}_{\text {semantic }}=3.51\right)$, frequency (the sum of the whole word frequencies for content words in the LEXESP corpus; Sebastián-Gallés, Cuetos, Carreiras, \& Martí, 2000; $\quad M_{\text {correct }}=469.50, \quad \mathrm{SD}_{\text {correct }}=431.84 ; \quad M_{\text {grammatical }}=519.32$, $\left.\mathrm{SD}_{\text {grammatical }}=692.04 ; \quad M_{\text {semantic }}=495.10, \quad \mathrm{SD}_{\text {semantic }}=611.83\right)$ or proportion of cognates $\quad\left(M_{\text {correct }}=.73, \quad \mathrm{SD}_{\text {correct }}=.26\right.$; $M_{\text {grammatical }}=.70, \quad \mathrm{SD}_{\text {grammatical }}=.29 ; \quad M_{\text {semantic }}=.70$, $\mathrm{SD}_{\text {semantic }}=.29$ ) (all $p s>.05$ ). Finally, 18 and 14 participants that did not take part in the fMRI study judged, respectively, the correctness of the grammatically violated sentences, and the plausibility of the semantically violated sentences. No accuracy differences were found in judging grammatical $(M=94.92 ; \mathrm{SD}=6.02)$ and semantic $(M=96.26 ; \mathrm{SD}=4.64)$ conditions $\left(t_{30}=0.88 ; p>.05\right)$.

For the task, the 40 correct sentences were used together with 40 incorrect sentences: 20 containing a grammatical violation and the rest containing semantic violations. We counterbalanced the material so that, from the anomalous sentences, those presented as grammatically violated to half of the participants were semantically anomalous to the other half, and vice versa. We added 40 strings of " $x$ " distributed as words within a sentence that matched the length of real sentences to complete the set (control condition); half of them presented "o"s between the "x"s, e.g. "xx 0000000 xxxx xx xxxxxxxxx".

\subsection{Procedure}

Before the scanning session, participants received the instructions and completed a practice block (with new stimuli not included in the experimental sessions). In this manner we ensured that they fully understood the task that they had to perform inside the scanner. During the scanning session, each participant completed a single scan consisting of 30 blocks of four sentences each (or " $\mathrm{x}$ " strings). In each block, correct and incorrect sentences where randomly included. There were 10 blocks for every condition: semantic violation, grammatical violation and control condition. At the beginning of every block, a screen with the word "SEMANTICA", "GRAMATICAL", or "IGUAL" appeared to indicate whether the sentences could include a semantic violation, a grammatical violation, or whether the strings of " $x$ " could include a string of "o", respectively. Each sentence was presented on a single line in black 24-point Arial font on a white background for $5 \mathrm{~s}$. and was preceded by a $1 \mathrm{~s}$. fixation cross. The participants responded pressing a button whenever they saw a sentence that included a violation (or the "x"s string included "o"s), and a different button when the sentence was correct (or the " $x$ "s string did not include "o"s). Blocks were pseudo randomized and lasted 24 s. each.

The stimuli were shown using the software Presentation ${ }^{\circledR}$ (Neurobehavioral Systems, Inc., Albany, CA, USA) and they were projected on goggles (Resonance Technology, Inc., Los Angeles, CA, USA) to the participants. Responses were obtained with a recording device Response Grip (Nordic Neurolab AS, Bergen, Norway). The presentation of the task was synchronized with the scanner via SyncBox equipment (Nordic Neurolab, Bergen, Norway). 
Table 1

Participants. Means (SD).

\begin{tabular}{|c|c|c|c|c|c|c|}
\hline Group & Age & AoA & $\begin{array}{l}\text { Self-reported L2 proficiency } \\
\text { comprehension }\end{array}$ & $\begin{array}{l}\text { Self-reported L2 proficiency } \\
\text { production }\end{array}$ & $\begin{array}{l}\text { Percentage of L2 use } \\
\text { (listening) }\end{array}$ & $\begin{array}{l}\text { Percentage of L2 use } \\
\text { (speaking) }\end{array}$ \\
\hline Bilinguals & $\begin{array}{l}23.30 \\
(3.59)\end{array}$ & 3 & $1.55(1.05)$ & $1.60(1.14)$ & $50.00(17.49)$ & $66.95(22.57)$ \\
\hline Monolinguals & $\begin{array}{l}25.10 \\
(4.48)\end{array}$ & - & $3.78(0.44)$ & $4.00(.00)$ & 9.25 (11.03) & $0(0)$ \\
\hline
\end{tabular}

Note: Self-reported proficiency punctuations range from 1 (very proficient) to 4 (non-proficient).

AoA = age of acquisition of Catalan.

\section{4. fMRI acquisition parameters}

Images were collected using a Siemens Avanto $1.5 \mathrm{~T}$ scanner (Erlangen, Germany) with a standard head coil. Structural 3D data were acquired using a T1-weighted gradient-echo pulse sequence $\left(\mathrm{TR}=11 \mathrm{~ms} ; \quad \mathrm{TE}=4.9 \mathrm{~ms} ; \quad \mathrm{FA}=10^{\circ} ; \quad\right.$ voxel $\quad$ size $\left.=1 \mathrm{~mm}^{3}\right)$. Thereafter we acquired 240 continuous EPI functional volumes $(\mathrm{TR}=3 \mathrm{~s} ; \mathrm{TE}=50 \mathrm{~ms} ; \mathrm{FA}=90 ; \mathrm{FOV}=224 \times 224 ;$ matrix $=64 \times 64$; voxel size $=3.5 \times 3.5 \times 3.5$; interslice gap $=0.5 \mathrm{~mm}$; 35 axial slices) parallel to the anterior-posterior commissure plane. Slices covered the entire brain.

\subsection{Imaging data analyses}

Image analyses were performed using SPM5 software (Welcome Department of Cognitive Neurology, University College London, UK). The first two volumes were excluded to avoid saturation effects. For each participant, the images were realigned to the first functional volume in the time series to correct head movements inside the scanner. None of our participants presented a movement greater than $3 \mathrm{~mm}$ between volumes. Following the realignment, the functional volumes were coregistered with the anatomical image and then the anatomical image was segmented. We used the parameters obtained in this last step to carry out the spatial normalization (Montreal Neurology Institute, MNI coordinates). Finally, the images were spatially smoothed with a Gaussian kernel (FWHM $=8 \mathrm{~mm}^{3}$ ). Time series were modeled and convolved using a canonical hemodynamic response function. The general lineal model was then applied to the analyses. We obtained contrast images for differences between experimental and control conditions for each subject and we included these contrast images into a random effects analysis to obtain group effects.

For each contrast image, we calculated a one-sample $t$-test to obtain population inferences for each group and compare both conditions (grammatical violation and semantic violation) within groups, as well as a two-sample $t$-test to compare activations between groups. To reduce the probability of false positives due to multiple comparisons (i.e. the concurrent testing of a large number of voxels in the brain), all results were thresholded at $p<.05$, family-wise error (FWE) corrected for multiple comparisons at the cluster level determined by whole-brain Monte-Carlo simulations (we used the program implemented in the REST software, Song et al., 2011; voxel-wise threshold of $p<.005$ and cluster-size criterion of 46 voxels). This procedure addresses the problem of multiple comparisons and has several advantages over single-voxel methods: single-voxel methods treat contiguous voxels as independent and neglect the fact that activated voxels belonging to the same region may be activated together (Ashby, 2011; Poldrack, Mumford, \& Nichols, 2011).

\section{Results}

\subsection{Behavioral results}

Behavioral data from 7 monolinguals and 2 bilinguals were lost due to technical problems in saving data to the computer. All the participants, however, found that the task was very easy (see accuracy in Table 2). The mean accuracy for the rest of the participants was included into a mixed analysis of variance (ANOVA), with Bilingualism (monolinguals vs. bilinguals) as a between-group factor and Type of trial (control vs. semantic decision vs. grammatical decision) as a within-participants variable. We observed a main effect of Type of trial, $F(2,30)=43.22$. Post-hoc tests revealed that responses to control trials were more accurate than to grammatical or semantic trials $(p<.01)$, and more accurate to semantic than to grammatical trials $(p<.01)$. The main effect of Group and the interaction of Type of trial $\times$ Group effects were not significant $(F s<1)$.

\subsection{Imaging results}

\subsubsection{Grammatical judgment}

3.2.1.1. Grammatical judgment vs. control. In bilinguals, the grammatical judgment versus control condition contrast (see Fig. 1, right) showed increased activation bilaterally in the IFG (BA 45) that extended to the dorsolateral prefrontal cortex in the MFG. Besides, in the left hemisphere, grammatical judgment was associated with increased activation in posterior brain regions: a big cluster peaking in the fusiform gyrus (BA 37) and extended to the cuneus and lingual gyrus in the occipital lobe (BA 18) and the cerebellum. In the right hemisphere, there was increased BOLD signal in the inferior parietal lobe (IPL) in the supramarginal gyrus that spread to the superior parietal lobe (SPL, BA 7). For the monolingual group, cortical activations were found in both the left IFG (BA 45/46/9), including pars opercularis, triangularis and orbitofrontal regions; the left superior frontal gyrus (SFG; BA 6), right and left cingulate gyrus (BA 8/32), and left fusiform gyrus (BA $18 / 23 / 37$ ). In addition, subcortical activation in the left thalamus and putamen, and in the right caudate was observed for this group along with regions in the right cerebellum (Table 3).

3.2.1.2. Between groups. Table 3 shows results of the contrasts in which grammatical judgment activations are compared between bilingual and monolingual groups. The bilingual group showed higher activation in the right superior and medial temporal gyrus (BA 22/21), and left IOG (BA 18/19) including the cuneus (Fig. 2). The opposite comparison (monolingual > bilingual group) did not show significant differences in the BOLD signal.

\subsubsection{Semantic judgment}

3.2.2.1. Semantic judgment vs. control (Table 4, Fig. 1). The semantic judgment versus control condition contrast in bilinguals exhibited recruitment of the left SFG (32/8), a large cluster peaking in the left

Table 2

Accuracy. Means (SD).

\begin{tabular}{llll}
\hline & \multicolumn{4}{l}{ Behavioral performance accuracy $(\%)$} \\
\cline { 2 - 4 } & Grammatical & Semantic & Control \\
\hline Monolinguals & $84.19(17.01)$ & $86.61(22.21)$ & $89.08(28.92)$ \\
Bilinguals & $87.11(9.30)$ & $94.75(4.30)$ & $96.29(6.25)$
\end{tabular}




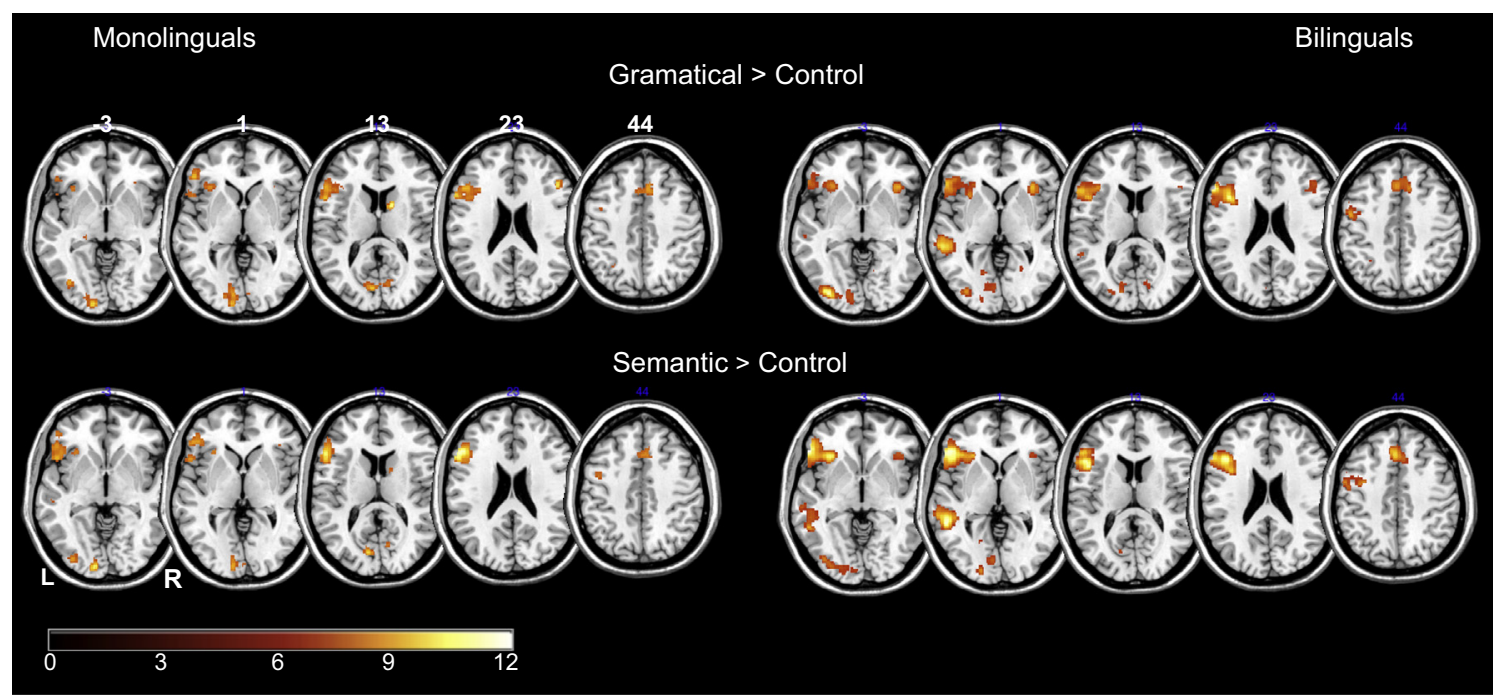

Fig. 1.

Table 3

Grammatical judgment vs. control.

\begin{tabular}{|c|c|c|c|c|c|c|c|c|c|}
\hline Contrast & Hemisphere & Brain area & Brodmann area & $x$ & $y$ & $z$ & $t$-score & $p$ & \#Voxels \\
\hline \multirow[t]{6}{*}{ Bilinguals } & Right & IPL & $40 / 7$ & 30 & -48 & 32 & 5.44 & .000 & 859 \\
\hline & & & & 30 & -50 & 48 & 5.16 & .000 & \\
\hline & & & & 36 & -46 & 44 & 3.99 & .000 & \\
\hline & Left & IFG & $45 / 44$ & -58 & 22 & 20 & 12.26 & .000 & 47,374 \\
\hline & & MFG & 9 & -42 & 12 & 24 & 11.70 & .000 & \\
\hline & & Fusiform gyrus & $37 / 18$ & -32 & -86 & -4 & 12.17 & .000 & \\
\hline \multirow[t]{6}{*}{ Monolinguals } & Right & Cingulate gyrus & $8 / 32$ & 2 & 18 & 50 & 10.56 & .000 & 2404 \\
\hline & Left & Cingulate gyrus & 32 & -12 & 22 & 30 & 3.07 & .003 & \\
\hline & & SFG & 6 & -2 & 2 & 64 & 9.06 & .000 & \\
\hline & & Fusiform gyrus & $37 / 23 / 18$ & -46 & -54 & -18 & 13.04 & .000 & 37,366 \\
\hline & & IFG & 45 & -40 & 16 & 26 & 12.50 & .000 & \\
\hline & & MFG & 9 & -42 & 10 & 32 & 11.58 & .000 & \\
\hline \multirow[t]{3}{*}{ Bil vs Mon } & Right & STG/MTG & $22 / 21$ & 56 & -30 & 2 & 3.21 & .001 & 71 \\
\hline & Left & IOG & $18 / 19$ & -46 & -80 & -10 & 4.14 & .000 & 81 \\
\hline & & & $18 / 19$ & -2 & -80 & 28 & 3.33 & .001 & 59 \\
\hline
\end{tabular}

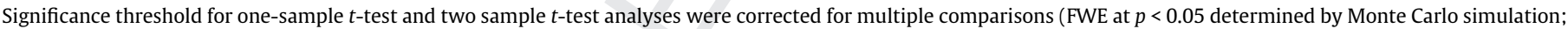

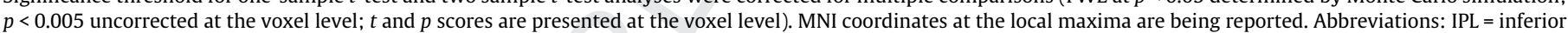
parietal lobule; IFG = inferior frontal gyrus; MFG = middle frontal gyrus; MTG = medial temporal gyrus; STG = superior temporal gyrus; IOG = inferior occipital gyrus.

middle temporal gyrus (BA 22) that includes the left IOG, the fusiform (BA 19/18) and lingual gyri (BA 18). The IFG (BA 45) was activated bilaterally. Activation was found in the right insula (BA 13). The monolingual group showed greater activation in a cluster in left IFG and a portion in the prefrontal cortex (BA 45/9), left medial frontal gyrus (BA 32/8), left precentral gyrus (BA 6), left insula expanded to the orbitofrontal region (BA 13/47), and the left cuneus (BA 17).

3.2.2.2. Between groups. Bilinguals revealed greater activation bilaterally, although more extended in the right hemisphere (Table 4). More specifically, bilinguals presented more activation than monolinguals while making semantic judgment in the left and right superior and middle temporal gyri (BA 21/22), right fusiform gyrus (BA 37), and left IOG (BA 19). Again, no differences in activation reached significance when monolinguals were compared to bilinguals.

\subsubsection{Grammatical vs. semantic judgment}

3.2.3.1. Grammatical vs. semantic judgment. Table 5 reports results comparing the functional activation between grammatical and semantic judgments. The monolingual group showed activation in frontal and posterior regions of the right hemisphere for the grammatical judgment compared to the semantic judgment, broader in the right hemisphere. More specifically, the right and left middle frontal gyri (BA 9), the left precentral gyrus (BA 6) and the right angular gyrus (BA 39/40) were involved. Activation in the right fusiform gyrus (BA 37), the lingual gyri (BA 19) and the right thalamus was also observed for the monolingual participants. No region was significantly more activated for the grammatical judgment compared to the semantic judgment in bilinguals.

3.2.3.2. Semantic vs. grammatical judgment. Comparing semantic and grammatical judgment, differences in activation in monolinguals were confined to the left hemisphere, in the medial (BA 9/8) and middle frontal gyrus (BA 10/9), the angular gyrus, and the inferior temporal gyrus (BA 20/21). Again, bilinguals did not present a greater activation for the semantic compared to the grammatical judgment in our task.

3.2.3.3. Between groups. When we compared bilinguals and monolinguals, only the grammatical vs semantic contrast presented significant differences. Monolinguals showed increased recruitment of brain tissue in right areas compared to bilinguals, namely the middle temporal gyrus (BA 21) and, subcortically, the thalamus, and hippocampus (Fig. 3). 
Table 4

Semantic judgment vs. control.

\begin{tabular}{|c|c|c|c|c|c|c|c|c|c|}
\hline & Hemisphere & Brain area & Brodmann area & $x$ & $y$ & $z$ & $t$-score & $p$ & \#Voxels \\
\hline \multirow[t]{9}{*}{ Bilinguals } & \multirow[t]{3}{*}{ Right } & IFG & 45 & 56 & 28 & 6 & 7.70 & .000 & \multirow[t]{3}{*}{5513} \\
\hline & & Insula & 13 & 40 & 24 & -4 & 7.38 & .000 & \\
\hline & & MFG & 46 & 46 & 20 & 26 & 6.76 & .000 & \\
\hline & \multirow[t]{6}{*}{ Left } & \multirow[t]{2}{*}{ SFG } & $8 / 32$ & -4 & 28 & 48 & 10.36 & .000 & \multirow[t]{3}{*}{511} \\
\hline & & & 8 & -4 & 18 & 52 & 9.39 & .000 & \\
\hline & & SMA & 6 & -2 & 4 & 66 & 6.94 & .000 & \\
\hline & & MTG & 22 & -52 & -44 & 4 & 12.61 & .000 & \multirow[t]{3}{*}{30,806} \\
\hline & & \multirow[t]{2}{*}{ IFG } & 45 & -54 & 30 & -2 & 11.93 & .000 & \\
\hline & & & 44 & -46 & 14 & 12 & 11.83 & .000 & \\
\hline \multirow[t]{9}{*}{ Monolinguals } & \multirow[t]{9}{*}{ Left } & \multirow[t]{3}{*}{ IFG } & $9 / 45$ & -56 & 20 & 26 & 15.27 & .000 & \multirow[t]{3}{*}{13,807} \\
\hline & & & 44 & -56 & 14 & 8 & 11.28 & .000 & \\
\hline & & & & -46 & 14 & 26 & 10.57 & .000 & \\
\hline & & \multirow[t]{3}{*}{ MeFG } & \multirow[t]{3}{*}{$8 / 32$} & 0 & 20 & 48 & 8.19 & .001 & \multirow[t]{3}{*}{196} \\
\hline & & & & -6 & 6 & 60 & 9.36 & .000 & \\
\hline & & & & -6 & 34 & 48 & 7.56 & .000 & \\
\hline & & Cuneus & \multirow[t]{3}{*}{$19 / 37$} & -38 & -84 & -10 & 12.54 & .000 & \multirow[t]{3}{*}{15,872} \\
\hline & & Fusiform gyrus & & -42 & -52 & -18 & 11.36 & .000 & \\
\hline & & Lingual gyrus & & -12 & -96 & 0 & 10.68 & .000 & \\
\hline \multirow[t]{5}{*}{ Bil vs Mon } & \multirow[t]{2}{*}{ Right } & Fusiform gyrus & 37 & 46 & -60 & -2 & 5.13 & .000 & 348 \\
\hline & & STG & $22 / 21$ & 60 & -36 & -8 & 4.24 & .000 & 342 \\
\hline & \multirow[t]{3}{*}{ Left } & STG & 22 & -58 & -2 & 4 & 3.84 & .000 & 69 \\
\hline & & MTG & $21 / 22$ & -68 & -34 & 4 & 3.33 & .001 & 55 \\
\hline & & IOG & 19 & -46 & -82 & -10 & 4.24 & .000 & 94 \\
\hline
\end{tabular}

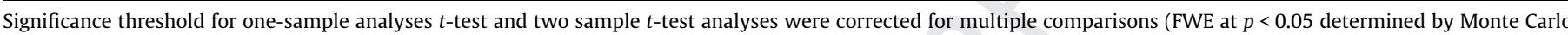

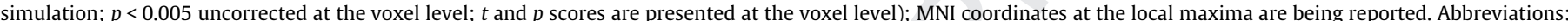

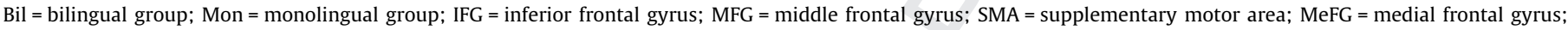
MTG = middle temporal gyrus; STG = superior temporal gyrus; IOG = inferior occipital gyrus.

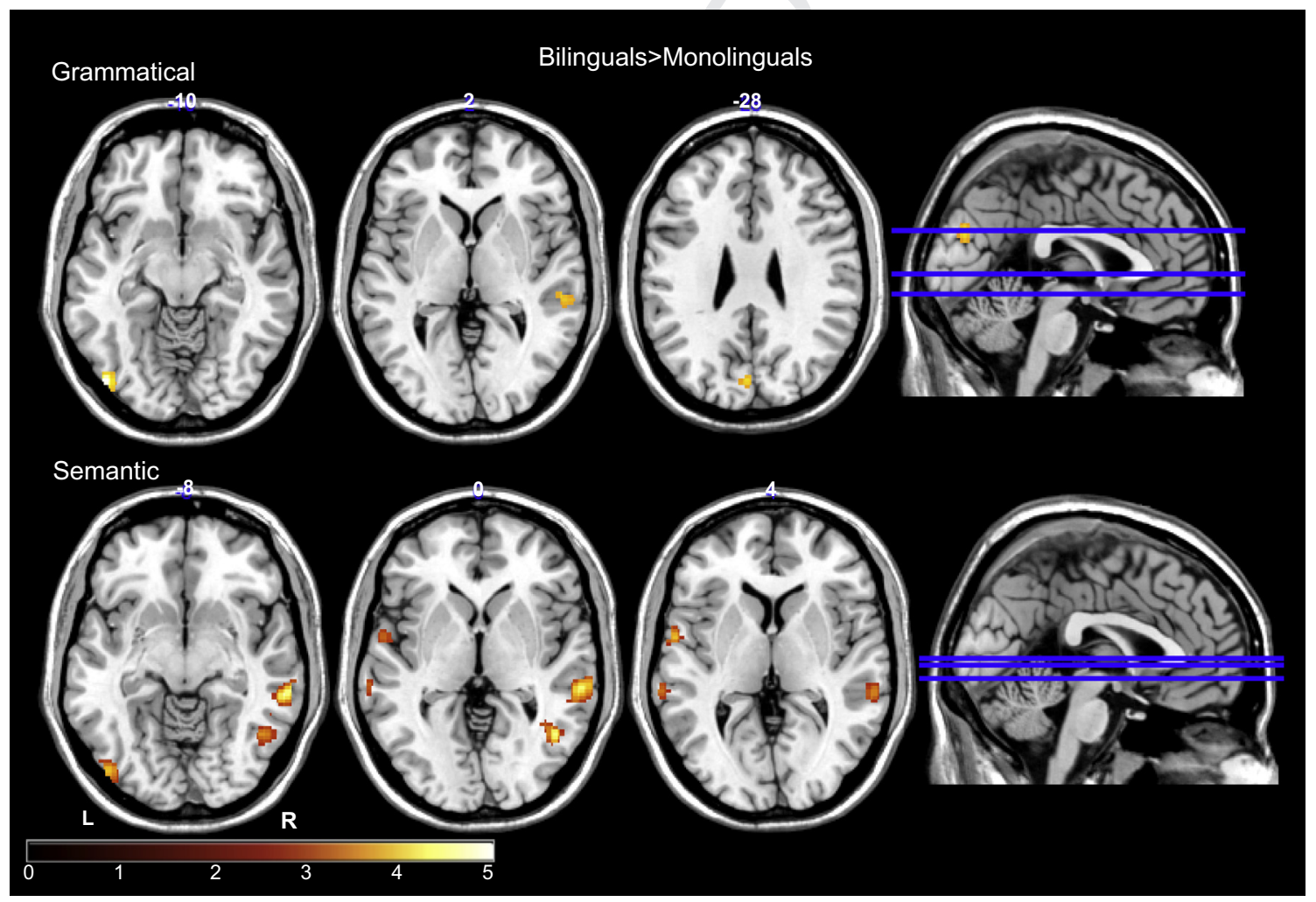

Fig. 2.

\section{Discussion}

The main purpose of the present study was to determine whether the pattern of activity associated with semantic and grammatical processing of sentences in their native language was different in early balanced bilinguals when compared to monolinguals. Both groups of participants performed two kinds of sentence verification tasks in the scanner based on, respectively, semantic and grammatical judgments upon exactly the same stimuli for bilinguals and monolinguals. Behavioral data showed that, even 
Table 5

Grammatical vs. semantic judgment.

\begin{tabular}{|c|c|c|c|c|c|c|c|c|c|}
\hline Contrast & Hemisphere & Brain area & Brodmann area & $x$ & $y$ & $z$ & $t$-score & $p$ & \#Voxels \\
\hline \multicolumn{10}{|c|}{ Grammatical judgment vs semantic judgment } \\
\hline \multirow[t]{12}{*}{ Monolinguals } & Right & Angular gyrus & $39 / 40$ & 32 & -56 & 38 & 9.65 & .000 & 8052 \\
\hline & & & & 28 & -48 & 42 & 7.26 & .000 & \\
\hline & & Supramarginal gyrus & & 44 & -44 & 42 & 7.15 & .000 & \\
\hline & & Fusiform gyrus & 37 & 50 & -56 & -10 & 5.48 & .000 & 623 \\
\hline & & ITG & & 52 & -60 & -18 & 4.85 & .000 & \\
\hline & & & & 58 & -50 & -8 & 4.81 & .000 & \\
\hline & & Thalamus & & 20 & -22 & 8 & 4.64 & .000 & 457 \\
\hline & & & & 10 & -26 & 8 & 3.85 & .000 & \\
\hline & & & & 16 & -24 & 0 & 3.25 & .001 & \\
\hline & & MFG & $6 / 9$ & 30 & -2 & 52 & 5.57 & .000 & 1918 \\
\hline & & & & 50 & 4 & 22 & 5.34 & .000 & \\
\hline & & & & 30 & 8 & 54 & 5.29 & .000 & \\
\hline \multirow[t]{3}{*}{ Mon vs Bil } & Right & Thalamus & & 16 & -24 & 2 & 4.32 & .000 & 204 \\
\hline & & MTG & 21 & 56 & -46 & -8 & 3.90 & .000 & 114 \\
\hline & & Hippocampus & & 28 & -14 & -22 & 3.31 & .000 & 71 \\
\hline \multicolumn{10}{|c|}{ Semantic judgment vs grammatical judgment } \\
\hline \multirow[t]{6}{*}{ Monolinguals } & Left & MeFG & $10 / 9 / 8$ & -10 & 54 & 18 & 6.44 & .000 & 1299 \\
\hline & & & & -20 & 60 & 28 & 5.69 & .000 & \\
\hline & & & & -8 & 36 & 58 & 5.39 & .000 & \\
\hline & & Angular gyrus & $39 / 19 / 22$ & -48 & -70 & 28 & 5.92 & .000 & 782 \\
\hline & & & & -40 & -62 & 22 & 4.01 & .000 & \\
\hline & & & & -48 & -64 & 16 & 3.88 & .000 & \\
\hline
\end{tabular}

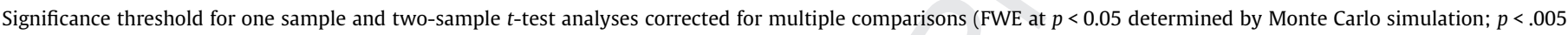

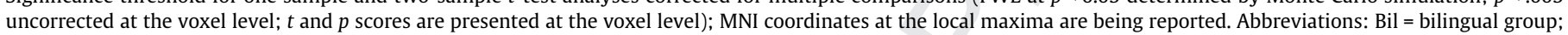
Mon = monolingual group; $\mathrm{MFG}=$ middle frontal gyrus; $\mathrm{MeFG}=$ medial frontal gyrus; $\mathrm{MTG}=$ middle temporal gyrus; ITG $=$ inferior temporal gyrus .

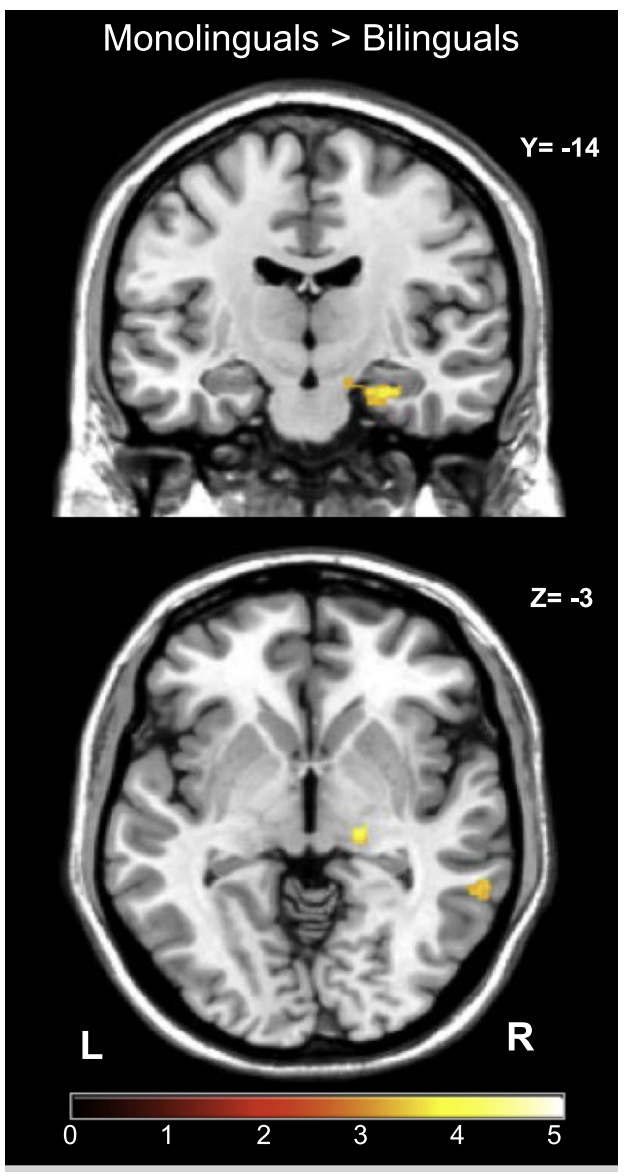

Fig. 3. of a sentence. Nevertheless, no difference in accuracy emerged between groups for any of the two tasks. Importantly, neural results showed that bilinguals activated the STG and other posterior language areas more than monolinguals, but no difference was found in crucial areas previously reported such as the IFG. Furthermore, monolinguals did not activate any brain area more than bilinguals in either semantic or grammatical judgment.

During both semantic and grammatical verification of Spanish sentences, the Spanish/Catalan bilingual group displayed more brain activity than the Spanish monolingual group. However, the opposite comparison did not yield differences; on average, no particular brain area in monolinguals showed a significant greater intensity of the hemodynamic signal than in bilinguals. Keeping in mind that accuracy of their behavioral performance was equivalent in both groups, our results are congruent with previous work showing a performance profile in bilinguals similar to monolinguals associated with more effortful processing in neural terms (for reviews see Abutalebi, 2008; Bialystok, 2009). Even in a language in which they are highly proficient, bilingual speakers recruit a greater extent of brain tissue in their native language than monolinguals (Kovelman, Baker, et al., 2008; Kovelman, Shalinsky, et al., 2008). Critically, our bilingual participants were highly fluent and proficient in Spanish, they acquired their L2 before the age of 4 , and the task included simple syntactic structures; still, they engaged some additional cortical areas while reading and processing Spanish sentences that Spanish monolinguals did not activate to the same degree.

When bilinguals judged if sentences were semantically plausible, their brains activated more than monolinguals' the bilateral STG, left middle temporal gyrus, right fusiform gyrus, and left IOG. Differences in left STG were previously found in a study comparing early bilinguals and monolinguals producing words in isolation (Parker-Jones et al., 2012), but not in comprehension (Rodríguez-Fornells et al., 2002). Sentence processing research reflects some contradictory results. Kovelman, Baker, et al. (2008) and Kovelman, Shalinsky, et al. (2008) found differences restricted to the IFG, but, using the same task and sentences, Jasinska and 
Petitto (2013), did find differences in the right STG between early bilinguals and monolinguals. The left posterior STG (pSTG) have been associated with phonological processing during reading tasks (Dietz, Jones, Gareau, Zeffiro, \& Eden, 2005; Graves, Grabowski, Mehta, \& Gordon, 2007; Jasinska \& Petitto, 2013; Jobard, Crivello, \& Tzourio-Mazoyer, 2003; Roux et al., 2012). The participation of this area in our study may be understood from the specific similarities and differences between Spanish and Catalan; both languages overlap significantly at the lexical level but differ in phonology. Then, the specific activation of left STG may be related to increased co-activation of different phonological representations in bilinguals when reading (Carrasco-Ortiz, Midgley, \& Frenck-Mestre, 2012; Haigh \& Jared, 2007). In this sense, it is important to note that increased co-activation in balanced and highly proficient bilinguals do not necessarily implicate a greater involvement of cognitive control processes during a task compared to low-proficient bilinguals or monolinguals. Cognitive control demands are normally higher in unbalanced bilinguals, where a dominant language interferes with a weaker language (e.g. Costa \& Santesteban, 2004).

Regarding the right STG, an increased activation was observed in bilinguals when processing both kinds of sentences (either in semantic or grammatical judgment) compared to monolinguals. Data in word production show activation in the right pSTG for late (Parker-Johnson et al., 2013) and early (Palomar-García et al., 2015) highly proficient bilinguals compared to monolinguals, but not in word comprehension. As Palomar-García et al. (2015) also point out, the activity in the right PSTG has been observed in association with a reduced vocabulary (e.g. Nagels et al., 2012) and bilinguals seem to have less word fluency in each language. More specifically, as we mentioned above, the Spanish/English bilinguals in the present study most likely use each language less frequently since they have reported to use Spanish and Catalan to the same extent every day. More importantly, although the studies above did not report differences between bilinguals and monolinguals in comprehension at the word level, Jasinska and Petitto (2013) found different involvement of the rSTG in sentence comprehension. With Jasinska and Petitto, we infer that the increased STG is a consequence of two languages co-activation in the bilingual brain. It is worth to mention that early bilinguals present structural differences in both left and right STG areas corresponding to the Heschl's gyrus compared to late bilinguals and monolinguals as seen recently (Berken et al., 2014; Ressel et al., 2012).

The IOG, a structure that - in our study - bilinguals recruited more than monolinguals in both tasks, is consistently activated in the processing of visual words (see Price, 2012). Research has demonstrated that low frequency words require IOG activity more than high frequency words, and pseudowords more than words. It seems, therefore, that difficulty in lexical access is involved in these differences and, thus, the differences in frequency of use for each language in bilinguals compared to monolinguals may be at the base of our findings here.

Contrary to previous studies in sentence comprehension using bilingual samples, we have not found any difference between bilinguals and monolinguals in the BOLD response in the IIFG. Even after lowering the threshold $(p<.10)$ the difference did not reach significance. Using complex sentences, Kovelman, Baker, et al. (2008) observed higher activation in IIFG for bilinguals; however, as mentioned before, Jasinska \& Petitto, could more precisely track this effect manipulating age of acquisition using the same task and material. More specifically, the IFG presented differences in late bilinguals (L2 acquisition at the age of 4-6) compared to early bilinguals (L2 acquisition at birth) and monolinguals, while differences between early bilinguals and monolinguals appeared in the right STG. Our study replicates this fNIRS result in early Spanish/Catalan bilinguals (L2 acquisition before the age of 4) in their native language and with simple sentences. In light of this outcome, with two groups that are matched in proficiency in their native language, our results are consistent with the proposal that the increased lIFG activation observed elsewhere in bilinguals most likely corresponds with increased demands in cognitive control due to competition between languages (Kim, Relkin, Lee, \& Hirsch, 1997; Marian, Spivey, \& Hirsch, 2003; Perani et al., 2003). Settings including both languages or even switching languages may specially heighten interference and competition between languages (e.g. Elston-Gütler et al., 2005; Palomar-García et al., 2015), which is a critical difference with the present study, where the language used during the whole session is Spanish. An explanation of greater IFG activation in bilinguals simply in terms of a greater co-activation (Jasinska \& Petitto, 2013) does not seem plausible, because the sample of bilinguals in our study belongs to a bilingual community that speaks two languages on a daily bases in a similar proportion and, in spite of this, they do not show increased involvement of IFG compared to monolinguals (see Table 1). Furthermore, our study points out a greater need to overcome interference in late bilinguals underlying IIFG effects in previous studies.

Our study also aimed to differentiate grammatical and semantic processing considering ERP and neuroimaging research, in which both kinds of processing show different correlates depending on bilingualism characteristics. To summarize, Wartenburger et al. (2003) showed that neural correlates during grammatical and semantic judgments in L2 were dependent on the age of acquisition and proficiency of L2, respectively. In the present study, monolinguals engage mostly frontal areas in grammatical processing compared to semantic processing and temporal regions in semantic compared to grammatical processing. Our findings are in line with other studies in monolinguals (e.g. Newman, Pancheva, Ozawa, Neville, \& Ullman, 2001). We observed differences between groups in the grammatical vs. semantic contrast. The monolingual group activated the hippocampus, MTG and thalamus when compared to the bilingual group. Such recruitment of temporal regions might be related to a deeper (semantic) processing of the sentences during the grammatical blocks for monolinguals (Clahsen \& Felser, 2006; Nyberg, 2002). The relatively higher load (concerning the co-activation of languages) in the bilingual group may prevent them from reaching the same level of encoding. Nevertheless, our judgment task cannot account for differences in the level of processing at the behavioral level between groups. Also it is important to notice that bilinguals did not show significantly different activation between semantic and grammatical processing at the present threshold. Since neither Warteburger et al. nor Rüschmeyer et al. directly compared between these conditions in their studies, there is no previous data to support our results. Further research is needed to shed some light in different group of bilinguals.

In summary, these results show that the Catalan-Spanish bilinguals in the present work activate the superior temporal gyrus, not the IIFG, more than monolinguals in a task that requires the use of only their native language. This suggests that even when early balanced proficient bilinguals perform linguistic tasks like sentence processing with similar accuracy as monolinguals, they recruit additional brain areas. However, these particular areas may be dependent on age of acquisition, languages usage, task conditions - type of stimuli and cognitive/linguistics demands - and, likely, the characteristics and the relative "similarity" between the languages that the bilinguals speak. It is possible that those aspects or features presenting high overlap between both languages in different levels of processing are more prone to cause differences in posterior language areas, whereas neural correlates in bilinguals, whose two languages almost show no overlap, are probably located in anterior language areas. 
Our data do not support a universal "bilingual signature" locked to a unique and invariable brain region, namely, the IIFG, - what we might name a strong hypothesis of neural signature of bilingualism - but they support a weak neural signature of bilingualism dependent of the particular conditions of processing associated with properties of bilingualism.

\section{Uncited reference}

\section{MacDonald, Cohen, Stenger, and Carter (2000).}

\section{Acknowledgments}

This research was supported by Research Grants PSI 2009-10067 and PSI 2014-47604-R (Ministry of Science and Innovation of Spain) and by BRAINGLOT, a Spanish Research Network on Bilingualism and Cognitive Neuroscience (CSD2007-00012, Consolider-Ingenio 2010 Scheme, Ministry of Science and Technology of Spain).

We would like to thank two anonymous reviewers for their invaluable comments that helped improve this work.

\section{References}

Abutalebi, J. (2008). Neural aspects of second language representation and language control. Acta Psychologica, 128, 466-478.

Ashby, F. G. (2011). Statistical analysis of fMRI data. Cambridge, MA: The MIT Press.

Berken, J. A., Mok, K., Chen, J., Gracco, V., Baum, S., Watkins, K., et al., 2014. Native bilingualism shapes the structure of Heschl's gyrus. Poster presented at the 44th annual meeting of the society for neuroscience. Washington, DC.

Bialystok, E. (2009). Bilingualism: The good, the bad, and the indifferent Bilingualism: Language and Cognition, 12, 3-11.

Bialystok, E., Craik, F. I. M., Green, D. W., \& Gollan, T. H. (2009). Bilingual minds. Psychological Science in the Public Interest, 10, 89-129.

Bialystok, E., Luk, G., Peets, K. F., \& Yang, S. (2010). Receptive vocabulary differences in monolingual and bilingual children. Bilingualism: Language and Cognition, 13, $525-531$

Carrasco-Ortiz, H., Midgley, K. J., \& Frenck-Mestre, C. (2012). Are phonological representations in bilinguals language specific? An ERP study on interlingual homophones. Psychophysiology, 49, 531-543. http://dx.doi.org/10.1111/j.14698986.2011.01333.x

Clahsen, H., \& Felser, C. (2006). How native-like is non-native language processing? Trends in Cognitive Sciences, 10, 564-570.

Clark, E. V. (2009). First language acquisition (2nd ed.). Cambridge: Cambridge University Press.

Colzato, L. S., Bajo, M. T., van den Wildenberg, W., Paolieri, D., Nieuwenhuis, S., La Heij, W., et al. (2008). How does bilingualism improve executive control? A comparison of active and reactive inhibition mechanisms. Journal of Experimental Psychology: Learning, Memory, and Cognition, 34, 302-312.

Costa, A., \& Santesteban, M. (2004). Lexical access in bilingual speech production: Evidence from language switching in highly proficient bilinguals and L2 learners. Journal of Memory and Language, 50, 491-511.

Dietz, N. A. E., Jones, K. M., Gareau, L., Zeffiro, T. A., \& Eden, G. F. (2005). Phonological decoding involves left posterior fusiform gyrus. Human Brain Mapping, 26, 81-93.

Elston-Gütler, K. E., Gunter, T. C., \& Kotz, S. A. (2005). Zooming into L2: Global language context and adjustment affect processing of interlingual homophones in sentences. Cognitive Brain Research, 25, 57-70.

Fiebach, C. J., Vos, S. H., \& Friederici, A. D. (2004). Neural correlates of syntactic ambiguity in sentence comprehension for low and high span readers. Journal of Cognitive Neuroscience, 16, 1562-1575.

Fine, I., Finney, E. M., Boynton, G. M., \& Dobkins, K. R. (2005). Comparing the effects of auditory deprivation and sign language within the auditory and visual cortex. Journal of Cognitive Neuroscience, 17, 1621-1637.

Friederici, A. D., Steinhauer, K., \& Pfeifer, E. (2002). Brain signatures of artificial language processing: Evidence challenging the critical period hypothesis. Proceedings of the National Academy of Sciences of the United States of America, $99,529-534$.

Garbin, G., Costa, A., Sanjuan, A., Forn, C., Rodríguez-Pujadas, A., Ventura, N., et al. (2011). Neural bases of language switching in high and early proficient bilinguals. Brain and Language, 119, 129-135.

Garbin, G., Sanjuan, A., Forn, C., Bustamante, J. C., Rodríguez-Pujadas, A., Belloch, V. et al. (2010). Bridging language and attention: Brain basis of the impact of bilingualism on cognitive control. NeuroImage, 53, 1272-1278.

Graves, W. W., Grabowski, T. J., Mehta, S., \& Gordon, J. K. (2007). A neural signature of phonological access: Distinguishing the effects of word frequency from familiarity and length in overt picture naming. Journal of Cognitive Neuroscience, 19, 617-631
Haigh, C. A., \& Jared, D. (2007). The activation of phonological representations by bilinguals while reading silently: Evidence from interlingual homophones. Journal of Experimental Psychology: Learning, Memory, and Cognition, 33, 623-644.

Jasinska, K., \& Petitto, L. A. (2013). How age of bilingual exposure can change the neural systems for language in the developing brain: A functional near infrared spectroscopy investigation of syntactic processing in monolingual and bilingual children. Developmental Cognitive Neuroscience, 6, 87-101.

Jobard, G., Crivello, F., \& Tzourio-Mazoyer, N. (2003). Evaluation of the dual route theory of reading: A metanalysis of 35 neuroimaging studies. Neurolmage, 20, 693-712.

Just, M. A., Carpenter, P. A., Keller, T. A., Eddy, W. F., \& Thulborn, K. R. (1996). Brain activation modulated by sentence comprehension. Science, 274, 114-116.

Kim, K. H. S., Relkin, N. R., Lee, K. M., \& Hirsch, J. (1997). Distinct cortical areas associated with native and second languages. Nature, 388, 171-174.

Kovelman, I., Baker, S. A., \& Petitto, L. A. (2008). Bilingual and monolingual brains compared: An fMRI investigation of syntactic processing and a possible "neural signature" of bilingualism. Journal of Cognitive Neuroscience, 20, 153-169.

Kovelman, I., Shalinsky, M. H., Berens, M. S., \& Petitto, L. A. (2008). Shining new light on the brain's "Bilingual Signature:" A functional Near Infrared Spectroscopy investigation of semantic processing. Neurolmage, 39, 1457-1471.

Kroll, J. F., Bobb, S. C., \& Hoshino, N. (2014). Two languages in mind: Bilingualism as a tool to investigate language, cognition, and the brain. Current Directions in Psychological Science, 23, 159-163.

Lenneberg, E. H. (1967). Biological foundations of language. Oxford: Wiley \& Sons.

MacDonald, A. W., Cohen, J. D., Stenger, V. A., \& Carter, C. S. (2000). Dissociating the role of the dorsolateral prefrontal and anterior cingulate. Science, 288, 1835-1838.

MacLeod, A., Fabiano-Smith, L., Boegner-Pagé, S., \& Fontolliet, S. (2013). Simultaneous bilingual language acquisition: The role of parental input on receptive vocabulary development. Child Language Teaching and Therapy, 29, $131-142$.

Marian, V., Spivey, M., \& Hirsch, J. (2003). Shared and separate systems in bilingual language processing: Converging evidence from eyetracking and brain imaging. Brain and Language, 86, 70-82.

Mayberry, R. I., \& Eichen, E. B. (1991). The long-lasting advantage of learning sign language in childhood: Another look at the critical period for language acquisition. Journal of Memory and Language, 30, 486-512.

Nagels, A., Kircher, T., Dietsche, B., Backes, H., Marquetand, J., \& Krug, A. (2012). Neural processing of overt word generation in healthy individuals: The effect of age and word knowledge. NeuroImage, 61, 832-840.

Neville, H. J., \& Bavelier, D. (2001). Variability of developmental plasticity. In J. McClelland \& R. Siegler (Eds.), Mechanisms of cognitive development: Behavioral and neural perspectives. Mahwah, NJ: Lawrence Erlbaum.

Neville, H. J., Coffey, S. A., Lawson, D. S., Fischer, A., Emmorey, K., \& Bellugi, U. (1997). Neural systems mediating American Sign Language: Effects of sensory experience and age of acquisition. Brain and Language, 57, 285-308.

Newman, A. J., Bavelier, D., Corina, D., Jezzard, P., \& Neville, H. J. (2002). A critical period for right hemisphere recruitment in American Sign Language processing. Nature Neuroscience, 5, 76-80.

Newman, A. J., Pancheva, R., Ozawa, K., Neville, H. J., \& Ullman, M. T. (2001). An event-related fMRI study of syntactic and semantic violations. Journal of Psycholinguistic Research, 30, 339-364.

Nyberg, L. (2002). Levels of processing: A view from functional brain imaging. Memory, 10, 345-348.

Ohnishi, T., Matsuda, H., Asada, T., Aruga, M., Hirakata, M., Nishikawa, M., et al. (2001). Functional anatomy of musical perception in musicians. Cerebral Cortex, $11,754-760$.

Ojima, S., Nakata, H., \& Kakigi, R. (2005). An ERP study of second language learning after childhood: Effects of proficiency. Journal of Cognitive Neuroscience, 17, 1212-1228.

Oldfield, R. C. (1971). The assessment and analysis of handedness: The Edinburgh inventory. Neuropsychologia, 9, 97-113.

Pakulak, E., \& Neville, H. J. (2011). Maturational constraints on the recruitment of early processes for syntactic processing. Journal of Cognitive Neuroscience.

Palomar-García, M. A., Bueichekú, E., Ávila, C., Sanjuán, A., Strijkers, K., VenturaCampos, N., et al. (2015). Do bilinguals show neural differences with monolinguals when processing their native language? Brain and Language, 142, 36-44.

Parker-Jones, O., Green, D. W., Grogan, A., Pliatsikas, C., Filippopolitis, K., Ali, N., et al. (2012). Where, when and why brain activation differs for bilinguals and monolinguals during picture naming and reading aloud. Cerebral Cortex, 22, 892-902.

Perani, D., \& Abutalebi, A. (2005). The neural basis of first and second language processing. Current Opinion in Neurobiology, 15, 202-206.

Perani, D., Abutalebi, J., Paulesu, E., Brambati, S., Scifo, P., Cappa, S. F., et al. (2003). The role of age of acquisition and language usage in early, high-proficient bilinguals: An fMRI study during verbal fluency. Human Brain Mapping, 19, 170-182.

Petersson, K. M., Reis, A., Askelof, S., Castro-Caldas, A., \& Ingvar, M. (2000). Language processing modulated by literacy: A network analysis of verbal repetition in literate and illiterate subjects. Journal of Cognitive Neuroscience, 12, 364-382.

Petitto, L. A., Zatorre, R. J., Gauna, K., Nikelski, E. J., Dostie, D., \& Evans, A. C. (2000). Speech-like cerebral activity in profoundly deaf people processing signed languages: Implications for the neural basis of human language. Proceedings of the National Academy of Sciences of the United States of America, 97. 13961-13966.

726 
Poldrack, R. A., Mumford, J. A., \& Nichols, T. E. (2011). Handbook of fMRI data analysis. Cambridge University Press.

Price, C. J. (2012). A review and synthesis of the first 20 years of PET and fMRI studies of heard speech, spoken language and reading. NeuroImage.

Ressel, V., Pallier, C., Ventura-Campos, N., Díaz, B., Roessler, A., Avila, C., et al. (2012) An effect of bilingualism on the auditory cortex. Journal of Neuroscience, 32, 16597-16601.

Roder, B., Stock, O., Bien, S., Neville, H., \& Rosler, F. (2002). Speech processing activates visual cortex in congenitally blind humans. European Journal of Neuroscience, 16, 930-936.

Rodríguez-Fornells, A., de Diego Balaguer \& Münte (2006). Executive control in bilingual language processing. Language Learning, 56(S1).

Rodríguez-Fornells, A., Rotte, M., Heinze, H. J., Nösselt, T., \& Münte, T. F. (2002). Brain potential and functional MRI evidence for how to handle two languages with one brain. Nature, 415, 1026-1029.

Rodríguez-Pujadas, A., Sanjuán, A., Ventura-Campos, N., Román, P., Martin, C., Barceló, F., et al. (2013). Bilinguals use language-control brain areas more than monolinguals to perform nonlinguistic switching tasks. PLoS One, 8, e73028.
Roux, F.-E., Durand, J.-B., Jucla, M., Réhault, E., Reddy, M., \& Démonet, J.-F. (2012) Segregation of lexical and sub-lexical reading processes in the left perisylvian cortex. PLoS One, 7, e50665. http://dx.doi.org/10.1371/journal.pone.0050665.

Rüschemeyer, S.-A., Fiebach, C. J., Kempe, V., \& Friederici, A. D. (2005). Processing lexical semantic and syntactic information in first and second language: fMR evidence from German and Russian. Human Brain Mapping, 25, 266-286.

Sebastián-Gallés, N., Cuetos, F., Carreiras, M., \& Martí, M. A. (2000). Lexesp. Léxico automatizado del español. Barcelona: Edicions de la Universitat de Barcelona.

Song, X.-W., Dong, Z.-Y., Long, X.-Y., Li, S.-F., Zuo, X.-N., Zhu, C.-Z., et al. (2011). REST: A toolkit for resting-state functional magnetic resonance imaging data processing. PLoS One, 6(9), e25031.

Wartenburger, I., Heekeren, H. R., Abutalebi, J., Cappa, S. F., Villringer, A., \& Perani, D. (2003). Early setting of grammatical processing in the bilingual brain. Neuron, 37, 159-170.

Ye, Z., \& Zhou, X. (2009). Executive control in language processing. Neuroscience $\mathcal{E}$ Biobehavioral Reviews, 33, 1168-1177. 